\title{
Human Genome Project
}

National Human Genome Research Institute (NHGRI)

\section{Source}

National Human Genome Research Institute (NHGRI). Human Genome Project.

The Human Genome Project was an international project that mapped and sequenced the entire human genome. Completed in April 2003, data from the project are freely available to researchers and others interested in genetics and human health. 